
allemande

47-1 | 2015

L'Allemagne et l'Europe | La déprise de l'Empire napoléonien en Allemagne en 1813

\title{
Günther SANDNER, Otto Neurath. Eine politische
}

\section{Biographie}

Vienne, Zsolnay, 2014

Geneviève Humbert-Knitel

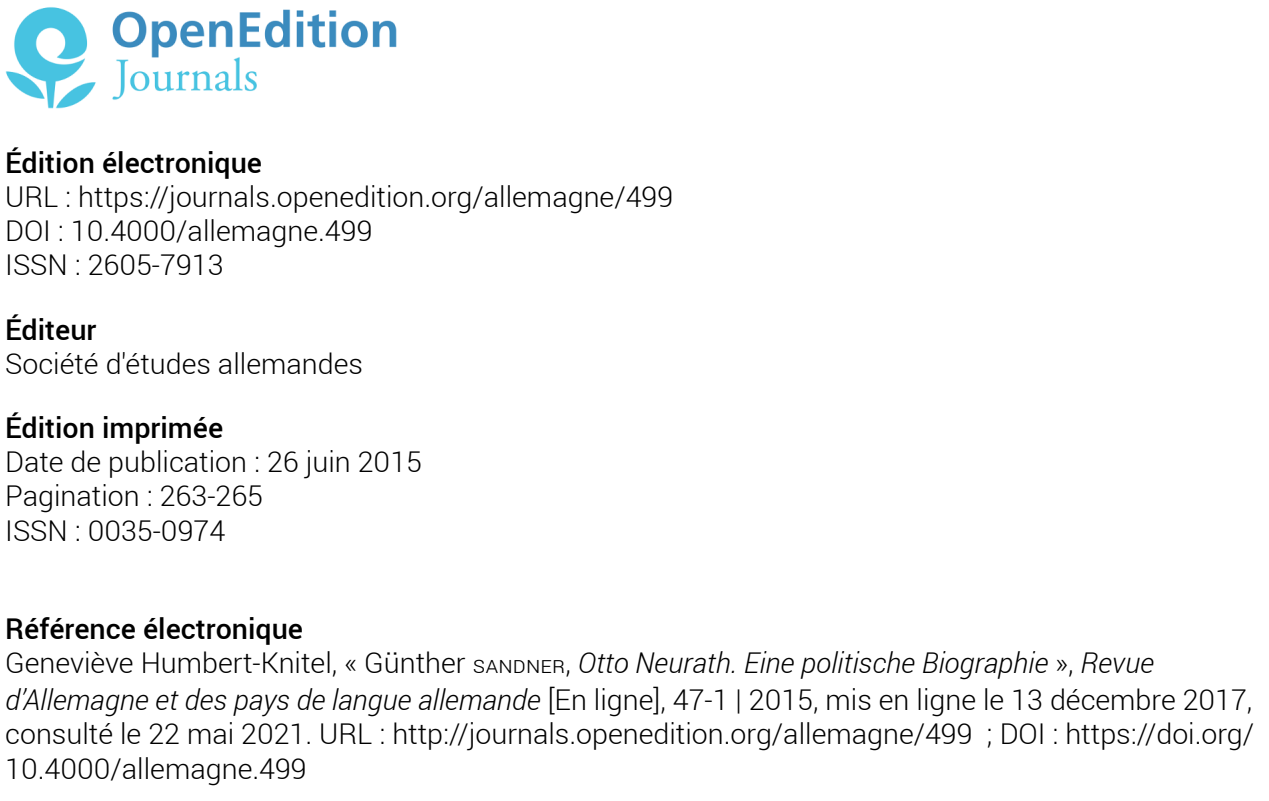

Revue d'Allemagne et des pays de langue allemande 
traumatisme qu'ils endurent. De la sorte, au-delà des précautions qu'imposent contexte de rédaction, orientation idéologique et dimension fictive de ce type de publications, c'est un livre qui en dit plus long sur l'évolution de la psychologie de son auteur et de ses camarades que sur l'événement lui-même. En ce sens, c'est un voyage au coeur des représentations individuelles et collectives pendant le conflit du côté de l'armée allemande que nous propose un ouvrage singulièrement instructif.

Stéphane LE BRAS

Günther SANDNER, Otto Neurath. Eine politische Biographie, Vienne, Zsolnay, 2014, $351 \mathrm{p}$.

Günther Sandner (né en 1967), qui enseigne les sciences politiques à l'université de Vienne, vient de publier une biographie à la fois politique et intellectuelle de Otto Neurath en montrant comment les événements politiques et sa conception du politique ont interféré avec ses nombreuses activités. Cette étude de 350 pages est écrite dans un style fluide, mêlant agréablement anecdotes et analyses en profondeur. Elle est accompagnée d'un appareil critique conséquent, une abondante bibliographie, un index des personnes bien utile et de nombreuses illustrations, et s'appuie sur un corpus de documents d'archives consultés en Autriche, aux États-Unis, en Grande-Bretagne, aux Pays-Bas et en Suède - autant de lieux qui témoignent d'une présence internationale et archivée de Otto Neurath. Autrichien né à Vienne en 1882 et mort en exil à Oxford en 1945, Neurath est difficile à classer. À la fois philosophe et figure de proue du Cercle de Vienne, il participe avec Rudolf Carnap à la rédaction du Manifeste de Vienne; réformateur social, économiste, urbaniste, il s'engage aux côtés de l'architecte Josef Frank dans la défense des citées jardins (Werkbundsiedlung) afin de rendre les vies humaines aussi heureuses que possible; planificateur, il conçoit en 1921 un plan d'économie planifiée pour la Palestine et, soutenu par la municipalité de "Vienne la Rouge ", il crée en 1925 un musée social et économique (Gesellschafts- und Wirtschaftsmuseum). Il en fut le directeur jusqu'en 1934, quand le régime dictatorial de Dollfuß l'oblige à émigrer aux Pays-Bas où il dirige le Mundaneum Institute, un lieu qui devait réunir toutes les connaissances du monde, une sorte de répertoire bibliographique universel. En 1940, avec l'arrivée des nazis, il émigre en Grande-Bretagne où, comme beaucoup d'Autrichiens juifs et non juifs, il est d'abord interné. Otto Neurath a laissé également son empreinte dans les sciences de la communication et du design et les sciences de l'éducation grâce à son combat pour la démocratisation du savoir avec ses travaux et ceux de ses fidèles collaborateurs, le graphiste Gerd Arntz et la dernière de ses épouses, Marie Reidemeister. II innova avec le langage par l'image, accessible à tous, avec des pictogrammes connus sous le sigle ISOTYPE, International System of Typographic Picture Education. Ces petites figures ou signes nous indiquent aujourd'hui le chemin à suivre ou nous aident à comprendre visuellement des statistiques et des chiffres souvent rébarbatifs, une sorte d'« esperanto de l'image », un langage qui se veut simple, universel voire transnational.

Otto Neurath était peut-être un génie universel ou, comme le dit son biographe, un " utopiste sceptique » qui concevait l'utopie comme la construction scientifique d'un monde possible, mais à coup sûr c'était une personnalité déroutante et dérangeante, de taille impressionnante, qui aimait signer ses lettres en dessinant des éléphants et qui se considérait comme un "scientifique du bonheur » (Wissenschaftler des Glücks). Si dans son introduction, Günther Sandner postule que Neurath était à la fois produit et acteur de son temps (p. 13) et que son histoire est une passionnante biographie intellectuelle de la première moitié du xxe siècle, alors comment expliquer le quasi-silence après sa 
mort en 1945 autour d'un homme dont la notoriété était à son apogée dans les milieux intellectuels et politiques de la Vienne de l'entre-deux-guerres? Dans les années 1970, Neurath est redécouvert en Autriche par les philosophes de l'institut du Cercle de Vienne qui lui consacrent de nombreux travaux, et aujourd'hui les milieux internationaux du design graphique reconnaissent largement l'influence qu'a exercée et qu'exerce encore la "méthode viennoise » sur leur production. Notons qu'en France, des journalistes du Monde diplomatique, entre autres les auteurs des Atlas du Monde diplomatique, tel que le géographe, cartographe et journaliste Philippe Rekacewicz, se réfèrent souvent à Otto Neurath pour expliquer leur approche politique et économique du monde (2).

Avec cette biographie, Günther Sandner comble un vide dans la recherche en offrant une synthèse inédite de la vie et de l'œuvre de Otto Neurath dans ses liens avec les sphères politiques, sociales et intellectuelles, non seulement en Autriche mais aussi au niveau international. La démarche est à la fois chronologique et thématique et retient cina grandes étapes dans la vie de Neurath: la première, en gros de 1901 à 1906, est celle des années de formation à Berlin, de ses rencontres et débats intellectuels en particulier avec Ferdinand Tönnies qu'il considérait comme son père spirituel, mais surtout avec les femmes, entre autres Ellen Key avec laquelle il débat d'éducation et de philosophie du bonheur, ou encore de Anna Schapire sa première épouse et grâce à laquelle il comprend que le combat des femmes, "die sogenannte Frauenfrage " ( $p .41)$ est un des grands enjeux du début du xxe siècle. En 1906, il soutient sa thèse de doctorat consacrée à l'histoire économique et sociale à l'époque romaine. Un des deux rapporteurs, Gustav Schmoller, déjà connu pour ses remarques antisémites, aurait dans son rapport souligné que le candidat Neurath était "Sohn eines Gelehrten, mit jüdischen Rasseneigenschaften » (p. 47-48). La deuxième étape se déroule de 1907 à 1918 et met en scène l'enseignant de l'économie de guerre (Der Kriegswirtschaftslehrer) à la nouvelle académie de commerce de Vienne, son goût pour les voyages d'étude en Galicie et en Bosnie et ses reportages sur les guerres dans les Balkans. Le lecteur retiendra que les analyses ne se limitent pas uniquement aux aspects économiques en période de guerre mais que Neurath, en véritable ethnologue, observe également les modes de vie et mentalités des populations rencontrées en notant par exemple avec humour que le "curieux peuple des Huzule " a coutume de mettre à disposition de l'hôte de passage la maîtresse de maison (p. 75). Durant cette période, Neurath cuvre activement au ministère de la Guerre à la mise en place avec Othmar Spann d'un comité scientifique sur l'économie de guerre dont les travaux devaient être conservés au musée de l'économie de guerre (Kriegswirtschaftsmuseum) à Leipzig et dont le directeur sera Otto Neurath. La troisième étape de ce récit de vie est consacrée à la courte période de l'immédiat après-guerre, de 1918 à 1919, marquée par les projets de réformes radicales économiques et sociales envisagées par les socialistes, tant en Allemagne qu'en Autriche. C'est sous la République des Conseils de Bavière que Neurath développera activement son grand projet d'utopie sociale de Vollsozialisierung, une sorte de vision communautariste voire collectiviste de l'économie et de la société devant assurer le bien-être des travailleurs. Mais la République des Conseils de Bavière ne dure qu'un temps et en mai 1919 Neurath est arrêté pour complicité de haute trahison et ne sera libéré que quelques mois plus tard et extradé en février 1920, grâce à l'intervention de Otto Bauer, à l'époque secrétaire d'État (c'est-à-dire ministre) des Affaires étrangères de la jeune république autrichienne. La quatrième étape de cette biographie, intitulée "Im Umfeld des Austromarxismus. Rotes Wien », couvre la période viennoise de 1920 à 1934, la période la plus politique mais aussi la plus féconde de Neurath, celle de sa collaboration avec les sociaux-démocrates de la ville de Vienne, tant sur le plan de l'urbanisme que de la démocratisation du savoir, avec la mise en place de sa méthode de visualisation des données statistiques (Bildstatistik, appelée

2 Dans le cadre de cette redécouverte et approfondissement de l'œuvre de Neurath, signalons la thèse en préparation de la germaniste Nepthys Zwer sur "L'ingénierie sociale de Otto Neurath» (Université de Strasbourg). 
Wiener Methode). Ici l'auteur ne laisse planer aucun doute sur les convictions politiques de Neurath en affirmant d'une part que dans les années 1920 et le début des années 1930 la "méthode viennoise " était pour Neurath essentiellement une arme de combat dans la lutte des classes (p. 190) et s'appuie sur les nombreux articles souvent polémiques que Neurath publia dans la presse de l'époque, qualifiée tantôt de marxiste tantôt de socialiste. Et comme pour confirmer sa thèse, Günther Sandner rappelle qu'au début des années 1930, Neurath a activement coopéré comme directeur du musée social et économique avec l'URSS à la mise en place à Moscou d'un nouvel institut de statistiques par l'image (Izostat), mettant ainsi la méthode viennoise au service de l'éducation politique et de la propagande soviétique. Cet épisode de la vie de Neurath est peu connu, comme le souligne Sandner, mais le lecteur aurait aimé en savoir davantage. Cette activité s'est déroulée dans le cadre officiel de la coopération austro-soviétique dont les buts, souligne l'auteur (p. 228), n'étaient pas politiques. Même si ce projet n'a pas abouti, il sauva néanmoins la vie à Neurath. En effet quand éclate les troubles de février 1934 à Vienne, il peut de Moscou via la Tchécoslovaquie s'exiler aux Pays-Bas où il restera jusqu'en 1940, quand avec l'arrivée des nazis, il s'exile en Grande-Bretagne. Sandner clôt ce chapitre par une phrase laconique: "Er sollte Österreich nie wieder betreten » (p. 232). La dernière étape de la vie de Neurath de 1934 à 1945 est celle d'un exilé politique qui survit grâce à ses réseaux antérieurs. Un événement de taille, son internement en Grande-Bretagne, aurait selon Sandner fortement marqué Neurath qui semble, au contact de ses compagnons de captivité, avoir découvert voire retrouvé ses racines juives. Jusqu'alors en effet, il ne se considérait pas comme juif puisque seul son père l'était. Sur le plan politique, Neurath conserva à Londres pendant la guerre de bons contacts avec les sociaux-démocrates autrichiens mais refusa toute coopération avec les communistes. Dans ses lettres écrites à son ami Josef Frank, l'économiste et sociologue Neurath fait un bilan critique de sa vie et de son action en faveur d'une économie planifiée basée non pas sur l'argent et la concurrence mais sur l'échange en nature (Naturalwirtschaft) et la coopération (p. 294). Mais il reconnaît que ce plan destiné à supprimer la pauvreté ne garantit pas forcément la liberté de l'individu qui, elle, n'est pas forcément acquise et pour laquelle il faut se battre.

En conclusion, l'auteur insiste sur l'actualité de la pensée de Neurath, en particulier en ce qui concerne ses efforts pour démocratiser le savoir, supprimer les privilèges de classe en matière d'éducation (p. 299), et Sandner a raison d'ajouter que les écrits de Neurath peuvent également être considérés aujourd'hui comme une critique avant la lettre d'une idéologie de la croissance sans limite, d'un capitalisme incontrôlé, "eine frühe Kritik an einer ungebremsten Wachstumsideologie » (p. 301). Une sorte d'hommage posthume à un « utopiste sceptique».

Geneviève HUMBERT-KNITEL

Claire TROJAN, L'identité interdite. Les expulsés allemands en RDA (1945-1953), Presses universitaires de Rennes, 2014, 354 p.

Claire Trojan donne, dans cet ouvrage tiré de sa thèse de doctorat, une vue d'ensemble bien ordonnée de la question des expulsés allemands en RDA, un sujet jusqu'à présent inédit en français, pour lequel elle s'est surtout appuyée sur les archives de l'Administration centrale des déplacés (Zentralverwaltung für Umsiedler ou ZVU, Berlin) et les fonds du Land de Saxe. Madame Trojan montre l'énorme défi social, économique et politique qu'a constitué l'arrivée de millions d'expulsés de l'est de l'Europe (un quart de la population de la Zone d'occupation soviétique en 1949) en puisant ses exemples jusqu'au niveau local (notamment la ville de Leipzig). L'ouvrage, qui comprend quelques illustrations et un glossaire, est 